\title{
KNOWLEDGE INCREASE IN THE F-ERA
}

\author{
Péter Kártyás
}

Abstract: F-Era is the era of freedom and flexibility. So in the era of Facebook a vast and continuously increasing amount of ideas and knowledge are available for those who are willing to search it. Access is easy, however, the quality of knowledge is questionable because it originates from passionate amateurs. The search for validated and relevant knowledge has become the main task and the evaluation of the results, which are available for the person longing for knowledge. It is the users' task and responsibility to decide if the available knowledge is of appropriate quality. If the browsing person, who seeks knowledge, is able to contextualize the new knowledge in accordance with the previous one, increase of knowledge happens. Today's organizations have realized the necessity of properly created knowledge management systems, and are looking for labour force which is competent, i.e. capable of interpreting its knowledge. The future of knowledge management is much rather about managing and teaching and training competent labour force for themselves within the framework of corporate universities.

Keywords: F-Era, knowledge increase, process of learning, knowledge management, competence, corporate universities

\section{Features of the F-Era}

The term 'F-Era' refers to many aspects of our present. On the one hand, based on the alphabetical order, it implies that in time we are after the E-Era, the e-tools and esolutions are no longer essentials, nor e-mail, e-commerce and e-learning are the most important keywords. On the other hand, ' $F$ ' stands for free, as in freedom. Freedom is of crucial importance in many fields of our lives just like free flow of capital, goods, labour force and knowledge but for us the freedom of learning and teaching is relevant. In the F-Era both learning and knowledge are available for everyone who has an Internet connection, and aims to obtain or share new knowledge. At the same time, F-Era refers to the Facebook phenomenon, as well, because the influence of social media on our everyday life is inevitable (Baracskai et al., 2014).

Facebook is important concerning our socialization too, posts and comments and shared contents are available in an abundance from all fields of life. Therefore, Facebook is not simply a tool for online communication among people but it has become a way for self-expression so most of us post emotions and thoughts, ideas and opinions on the world on a daily basis. Even if not all of the users of Facebook use the site to share their inner world but almost all of the users read the posts of their friends and acquaintances. It is important to realize that Facebook functions as a fake mirror because most people are trying to show a better and healthier picture of themselves than they really are. This distortion appears in many aspects of our lives and drives us to always question the reliability of information read on Facebook. 
Nowadays Facebook is often considered as a source of news and information on interests, and most of us browse it to update their actual knowledge about the events and things of the world. However, most sources of information are usually trusted by the person seeking knowledge, it is still the reader's responsibility to choose the relevant sites, news portals or blogs to avoid fake or misleading knowledge which is not validated at all. If a person or organization is followed, the follower has to take it into consideration that the quality of the content shared is uncertain and has be aware of the fact that it is not necessarily true or validated. So in the Era of Facebook a vast and continuously increasing amount of ideas and knowledge is available for those who are willing to search it. Access is easy, however, the quality of knowledge is questionable because it originates from passionate amateurs (Anderson, 2008). The search for validated and relevant knowledge has become the main task and browsing and surfing the net and the evaluation of the found results which are available for the person longing for knowledge. It is the users' task and responsibility to decide if the available knowledge is of appropriate quality. If the browsing person, who seeks knowledge, is able to contextualize the new knowledge in accordance with the previous knowledge, increase of knowledge happens.

F-Era is the era of flexibility, too. Nowadays it is more complicated for the rest of us to maintain attention for a longer period of time because so many stimuli reach us in every minute. Carr says that reading is a different thing as it used to be in the past because books are often replaced by online contents (Carr, 2010). Back in the days reading meant continuous focusing and while we read our attention was not driven away by other things. At present, reading online texts is not simply reading any more, it became browsing because whilst reading the reader may meander.

Texts of simple articles on news sites can often take us to new homepages, direct links make it possible to jump to other texts and other online contents whilst in the past a link meant only footnotes or the reference. This network of online content is very tempting for most of the users so a larger amount of time is spent on the Internet with browsing, reading, following related links of the topic even watching attached videos. When searching for new knowledge, a chance is given to dive deeper in a topic, to check all previous articles or papers published concerning our interest, many online tools such as dictionaries or translation supporting sites are available. This whole system of abundant information seems to foster a better understanding of the knowledge found but there are so many details that usually less is more so we have to be able to shift of focus. Flexibility, the ability to change our focus quickly has become a new aspect of learning, searching and gathering knowledge.

At present the economic significance of Facebook is inevitable, too, as it is a main platform for organizational communication and online marketing. For most enterprises presence on Facebook is essential to present itself for the stakeholders. The online success of a business can be calculated based on the number of likes and comments or the number of its followers of its Facebook profile. However, a liked profile or a product's profile will not result in any profit but this information can be used to estimate the number of customers the enterprise can achieve (Godin, 2008). The key of the Facebook phenomenon is presence. One and a half decade ago we could say that an enterprise is viable only if it has a homepage on the Internet but by now this same minimal requirement is connected to the appearance on Facebook and other social media sites. 


\section{The Importance of Knowledge Management in the F-Era}

The importance of knowledge is no longer a question for any organizations. Our world has become totally globalized, and by the technical conditions provided by the Internet communication and the trading of information happens faster than ever. Geographical distances do not hinder persons and organizations to contact each other quickly, the sharing of knowledge has become easy due to digitalization. As a consequence of the facts mentioned above, global economy has changed, too, and by now a much bigger emphasis is laid on knowledge based sectors, and this proportion is still growing. Such classical assets as capital or labour seem to be replaced by knowledge possessed by labour and instead of financial strength knowledge has become the the source for competitive advantage for even non competing organizations like nonprofit and government institutions.

Most organisations have realised that the key to its success is the proper management of knowledge as by now it has turned out to be the most crucial resource. If an organisation is able to manage knowledge in an appropriate manner, it can contribute to such strategic result as profitablilty, competitiveness and capacity enhancement (Jeon et al., 2011). In the past decades the topic of knowledge management has become really popular with both HR experts, philosophers and researchers of social studies. The management of knowledge is considered as an essential factor for organizational sustainability and the maintenance of competitive advantage. Knowledge management is often defined as a complex system for an organization's strategy, structures and processes so that the organization can apply its knowledge to create both market and social value for its stakeholders. Organisations require a strong capacity to obtain, develop, organise, and sustain their employees' capabilities in order to be able to compete its competitors. Organisations that effectively manage and transfer their knowledge are more innovative and perform better (Riege, 2007).

Most organisations, which by now have understood why they must manage knowledge, create concepts how to achieve their objectives and dedicate time and energy to these efforts. Knowldge management has been described as a key driver of organisational performance (Bosua and Venkitachalam, 2013). So managing and utilizing knowledge effectively is crucial for the organizations to take full advantage of the value of knowledge. The attention and importance given to knowledge in literature as well as practice in the past decade is also of necessity due to changes in the operating environment such as the increasing globalization of competition, speed of information transfer and the acceleration of aging of knowledge, and the dynamics of innovations (Greiner et al., 2007). In a knowledge based economy, knowledge management is increasingly considered as critical for an organization to be effective and drive performance.

Knowledge management in the F-Era is necessary for leaders at all levels of an organization to be considered as a prerequisite for higher performance and flexibility in both private and public sector. For a couple of years, knowledge management was believed to be just another fashionable management tool that leaders are willing to add to their toolbox to impress stakeholders but with the passage of time, this will fade away, too, as many others (Scarbrough and Swan., 2001; Ponzi and König., 2002; Hislop, 2010.; Serenko et al., 2010; Oluikpe, 2012). Since the concept of knowledge management emerged in the literature at the end of the '90s many academic papers have been published concerning the topic, whose number is still increasing, so by now the belief that this was a whim only has been proven false. 


\section{Concepts on Knowledge}

Based on the work of Blackler five different forms of knowledge can be separated. So knowledge can be embodied, embedded, embrained, encultured, and encoded (Blackler, 1995). Blackler defines embodied knowledge as knowledge that is gained through training of the body to perform a task, which also implies that knowledge cannot be independent from human activity, and knowledge will always be strongly attached to human beings' body.

The next category of knowledge defined is embedded knowledge, which is knowledge that is found in routines and systems. Everyday tasks in an organization, daily routines or the ways people think and feel about their jobs, can hold embedded knowledge as the habits foster learning among the workers that are beyond their job tasks. So embedded knowledge is inseparable from work practice and also embodied by the employees who manage these practices (Strati, 2007; Yakhlef, 2010). For example, an employee cleaning the corridors of the company headquarters, relies on his everyday routines, movements and practices using embedded knowledge whilst the sum of all the activities he does represent embodied knowledge because the person needs his body to perform a task even when he does not move at all, just stand and think on the next steps of the task.

Embrained knowledge is defined as the knowledge that a person can possess but has difficulties expressing in words or sharing with others. It is also can be described as knowledge that one cannot easily write down, talk about with others, or represent with pictures or other tools. This type of knowledge is gained through experiencing and may reflect one's perceptions, opinions, values and morals. Compared to the categories presented above it can be said that embrained knowledge is always embodied but not necessarily embedded, too. Sticking to the previous example, the janitor can apply embrained and embedded knowledge during his work when he uses special movements for washing the floor because it cannot easily be told to a younger colleague how to perform it quickly and effectively, and it is a part of the workers routine. Or it is a quite similar situation that he neither can explain to his colleague how to court a woman because this is a type of knowledge which can be learned only by experiencing and explanations on this topic are both difficult to give and useless in practice. On the other hand, this knowledge is not embedded in our example because our janitor rarely courts women, so it is not a routine or system in his life. Still worth thinking through how could this type of knowledge be considered in case of a young, handsome man who is really experienced in socializing and often dates women.

Encultured knowledge is described as a set of knowledge that is shared among groups of people who share a similar environment or culture. If a group of workers all share an understanding what kinds of behaviour are accepted within the organization, or what actions and beliefs are considered normal, it can be said, that they have the same encultured knowledge. Encultured knowledge is always embodied, can be embedded and sometimes embrained too. The janitor knows the rules of social interactions within the company, can explain to his younger colleague how to welcome the leaders of departments or the white collar workers (encultured, embedded, but not embrained knowledge) but not necessarily can explain the significance of the company's mission statement even if he knows and understands it (encultured, not embedded, but embrained knowledge).

Encoded knowledge is a form of knowledge that can be easily written down, expressed in words or pictures, and is transferrable in many ways. Examples of it are 
procedure manuals, guidelines, process diagrams or maps, charts and sheets on instructions, or recipes of encoded knowledge because they are encoded in a physical form that is understandable by a lot of people. The janitor surely knows or can easily access the basic documents regarding his work like the collective agreement, he is familiar with the logo of the company, knows the documents concerning health and safety or the rules of work laid in guidelines and manuals.

Thinking of organizational knowledge in general it can be stated that it is embodied and embrained in the employees, embedded in everyday routines and common tasks, encultured among the staff, and encoded in manuals, regulations, guidelines and standard processes. Davenport and Prusak says that in organizations, knowledge becomes embedded not only in documents but also in organizational routines, processes, practices, norms and cultures so it can be defined as the sum of all intellectual assets that can be found within an organization (Davenport and Prusak, 2000). It is embedded knowledge which is found primarily in relationships among employees of the company and in behavioral norms, beliefs and ways of making decisions that affect their relationships with each other.

According to another point of view knowledge can also be distinguished in two different types. Polanyi describes knowledge as existing in two main dimensions as tacit and explicit knowledge (Polanyi, 1967). Knowledge is most commonly referred to as either explicit (encoded) or tacit (which is in humans' heads). Tacit knowledge is always personal and context specific knowledge of a person so it is within the human mind. It evolves from people's interactions and requires skill, practice and experience. Therefore, tacit knowledge is highly personal, subjective, difficult to explain, express or communicate entirely, it is experience based, context and job specific, transferred through conversation or narrative, not captured by formal education or training and may even be subconscious but capable of becoming explicit knowledge (Nonaka and Takeutchi, 1995). It is the type of knowledge that is used mostly by employees of an organizational when performing tasks. Tacit knowledge is hard to tell in words because it is expressed through action based skills and on the job activities and cannot be simplified to rules, exactly defined processed and sequences. So tacit knowledge is reflected in actions, executed tasks, procedures, commitment, values and beliefs.

Referring back to Blackler's concept, tacit knowledge is embrained knowledge so if its explication requires the use of metaphors and an extensive process of socialization. Sharing of tacit knowledge is made possible through networking among those who possess it and this is referred to as Communities of Practice $(\mathrm{CoP})$. It is good to be aware of the fact that tacit knowledge is difficult to copy or steal by competitors so this makes it an important source of sustainable competitive advantage. Furthermore, the major challenge for organizations is to develop strategies which make possible the transformation of the tacit knowledge into explicit knowledge.

Explicit knowledge, on the other hand, is formalized and systematic; it can be codified, collected, stored, and disseminated throughout the organization. It is not bound to a single person and is similar to the specialties of data. Explicit knowledge can be objected to sharing by information technology. Explicit knowledge has many examples as it can appear in tangible forms of books, journals, manuals, sheets, guidelines, illustrations, pictures, geographical or process maps, blueprints, technical specifications, mathematical formulas, visualized charts and diagrams. It can simply be documented or expressed in words or numbers, and shared formally. Due to all these characteristics listed above, explicit knowledge can easily be managed by organizations. Polanyi 
distinguishes between tacit and explicit knowledge by suggesting that it is possible for people to know more than they can tell.

Explicit knowledge is the part of tacit knowledge that can be expressed by words and does not represent the entire knowledge. While tacit knowledge can be possessed on its own, explicit knowledge must be based on being tacitly understood and applied, therefore all knowledge is either tacit or rooted in tacit knowledge. A good example of explicit and tacit knowledge could be a guidebook on how to play the popular card game, poker. Explicit knowledge used in the game is which consists of the presentation of the deck, individual illustrations of cards (clubs, diamonds, hearts, spades) used during the game, or the rules, which explain what actions and plays are allowed, or how worthy the combinations of cards are. Tacit knowledge is an understanding of what makes a good hand, how to quickly shuffle the deck or particularly how and when to bluff during the game or not. The processes such as making decisions on raising the bet or to quit, observing the other players' reactions and making assumptions on their capabilities concerning bluffing are often difficult to express in words.

Dividing of knowledge into explicit and tacit is rather too simple, so a new category can be added by stating that knowledge is better described as explicit, implicit, and tacit. Explicit means information or knowledge that appears in tangible form. Implicit is information or knowledge that is not set out in tangible form but could be made explicit while tacit knowledge that one would have extreme difficulty operationally setting out in tangible form. On the other hand, some researchers categorize organizational knowledge into tacit, explicit and cultural parts. But whether tacit, implicit, explicit or cultural, the most obvious point is the making of the organization's data and information available to the members of the organization.

Independently from the categorization of dimensions of knowledge, the process of knowledge transfer is of crucial importance. Knowledge appears as a result of an interaction of explicit and tacit knowledge, and the process of creating knowledge results in continuous increase of personal knowledge. The process of knowledge increase begins with persons sharing their internal, tacit knowledge by contacting with others or by capturing it in written form. Some people then internalize the shared knowledge, and that process creates new knowledge. These people with the newly created knowledge then share this knowledge with others and the process begins again.

Because knowledge is basically tacit and owned by individuals it is difficult to gain control over it. Organizations need to formalize and store the individual's knowledge to be able to use knowledge more efficiently. This means that organizations have to make tacit knowledge explicit and make it available for all other members by spreading knowledge across the organization so it could become a part of organizational knowledge. These transformation processes are highly supported by information and communication technologies. Therefore, the main focus task an organization has to deal with transforming tacit knowledge into implicit then explicit knowledge, and assure that individual knowledge becomes organizational knowledge. This process must be properly and thoroughly planned and managed by all organizations that aim to become successful, sustainable, competitive firms with the need of being truly innovative and learning organizations built on professional knowledge sharing culture.

\section{Components of Knowledge Management}

Due to the spreading of theories on knowledge and knowledge management many organizations have realized that the most important and sustainable competitive 
advantage they have is the well trained and continuously developing labour labour force so they must lay a significant emphasis on knowledge management if they are to maintain efficient operation. Such a company must have balanced capacity for obtaining, developing and organizing their employees' competencies. The realization came that processes and technology alone are not enough to drive an outstanding performance and human resource has a vital role in an organization's success. To be able to comply with the dynamically increasing challenges of the globalized economy and markets companies must develop a strategic approach for the management of knowledge, and to this end, serious attention must be paid to four key components: Knowledge, People, Processes and Technology (Desouza, 2001). In other words, the main role of knowledge management is to build bridges between people, processes and technology to achieve the goal of capitalizing on knowledge.

Knowledge is described as an essential part of knowledge management. Without having knowledge to manage there would be no knowledge management. Knowledge basically refers to a collection of information. This could mean that the information is embedded in the form of theories, processes, systems, or it could be voiced in the form of opinions, theories, ideas and analysis. As it was presented previously different typologies have been developed for the categories of knowledge but the only consensus is the notion that knowledge is more than simply data and information. Davenport and Prusak define knowledge as a fluid mix of framed experience, values, contextual information and expert insight that provides a framework for evaluating and incorporating new experiences and information (Davenport and Prusak, 2000). It originates from and applied in the minds of knowers'.

The second component of knowledge management is people. All knowledge is rooted in people which in the previous chapter was referred to by the term 'embodied' i.e., without humans there would be no knowledge at all. The ability of humans to think creatively and individually mixed with experience and talents make humans valuable sources of knowledge. People are both the creators and consumers of knowledge because individuals obtain knowledge from various sources every day just like they create knowledge. All in all, it can be said that the management of knowledge requires the presence of people at all the stages of the process. Consequently, it is a must to consider people as key element in knowledge management strategy and implementation. People face emergent knowledge needs as part of daily work tasks. And these needs should be fulfilled through tools, processes, systems and protocols to apply relevant knowledge.

Baloh et al. define processes, which is another component, as mechanical and logical artifacts that guide how work is conducted in organizations (Baloh et al., 2011). Processes direct work and are critical to the functioning of an organization. Processes can consist of, and executed by humans, machines or a combination of these. A crucial requirement for knowledge management is to be able to overview operating processes and how to map them. If the inputs, outputs, personnel, resources and work being conducted are known, a given process can be easily described. Process mapping can help to visualize what is happening in the organization and how strategy is being executed by managing consecutive tasks. The precise level of knowledge and competence required to accomplish steps and tasks of the business process can then be determined, and requisite technology or human intervention can be assigned to meet the needs with the goal of maintaining effectiveness and efficiency.

The last component of knowledge management is technology. The support provided by information and communication technologies is vital for effective 
knowledge management. Modern IT technologies can foster cooperation between people and teams which are geographically located far from each other. It can also be used to support activities through the codification of knowledge just like vivid and interactive forms of communication through the Internet. Even though technology is important, helpful and can significantly back up knowledge management, it is necessary to notice that it is not a solution on its own. Technology is simply a great tool but without the commitment and expertise of people for implementing knowledge management system it will not make any organization share knowledge or gain the effectiveness and competitive advantages of it. If the leaders of the organization truly believe in the benefits of a highly performing knowledge management system, technology can significantly contribute to its success.

\section{Change in the Process of Learning}

As it was presented in the first issue, one of the most important features of the FEra is the unlimited quantity of knowledge available. The consequence of this abundance is that knowledge is shallow and its validity and quality are questionable. The knowledge acquired during the traditional educational system is validated and approved, which means that in the past, then and there, it worked under certain circumstances. On the other hand, this kind of knowledge is not really valuable in the F-Era because it is easily accessible for everyone and quite surely refers to the past. So it is not necessarily valid in the present and will surely be outdated by tomorrow.

The essence and process of learning was described in different ways by the different disciplines of psychology. Behaviorism defines it as a changing of our responses to stimuli. According to the views of cognitivism learning is a process of storing, managing and recalling memories. The constructivism approach of pedagogy says that learners create knowledge and meaning themselves in order to understand the world around so learning happens by reconstructing known terms and experiences and their connections about the world. It is important to notice that in this way the knowledge increase is not a cumulative process, new knowledge is not simply added to the previous experience but new elements of knowledge adjust the previous ones, have an effect on it so the process results in reconstruction and reorganization. At the beginning of the learning process the learner had a bit misty picture of the world, and the essence of learning is that after the process a new, clearer, sharper, a more detailed and more elaborate picture will emerge in a different way. The learner gives meaning to the new knowledge in accordance with the previous knowledge and rules of thinking but the new knowledge also affects the organization of the previous picture, it reforms it so a new big picture is formed by learning.

Based in Polanyi's work, we can say that when we learn we build our personal knowledge (Polanyi, 1962). According to the philosophy of constructivism, personal knowledge is built as a result of a reconstructing process, which is individual, affects the person who is learning and lasts for the entire life of the learner. So the term of lifelong learning refers to all people, not only for those who learn in a formal way in the traditional educational system even throughout their whole life or always search for new topics and knowledge to learn. This approach changes the picture of traditional education because it says, that it is not the teacher who transfers his knowledge to the student so there is no intermediary in the process but a process of reconstruction happens within the mind of the student. So during the process of learning not simply new knowledge is added to the old one but the entire personal knowledge changes through 
reconstruction and it can be said that during our lives, our knowledge is in continuous transition.

\section{Organizational Expectations on Knowledge and Competence}

There are a lot of important factors that emphasize the need for effective knowledge management like the survival of the organization, the impacts of globalization or the aging of the labour labour force. Considering the management dynamics nowadays, the needs of managing knowledge requires tense attention because most of the work is data- and information based. Most organizations independently of operating in the private or public sectors compete on the basis of knowledge because both products and services are becoming increasingly complex As a consequence, lifelong learning has become an inevitable necessity and knowledge management has gained more importance than even a decade ago because marketplaces are increasingly competitive and the rate of innovation is constantly rising.

Retiring and fluctuating staff also creates a need to replace informal knowledge with formal, standardized methods and processes. It is also of importance because early retirements and increasing mobility of the labour force lead to loss of knowledge while changes in strategic direction may result in the loss of knowledge in some specific areas. It must be realized that due to the faced working environments knowledge and information have become a field where new business problems occur. Consequently, managing knowledge gives an opportunity for organizations to achieve higher effectiveness, apply significant improvements in human performance and competitive advantage.

Desouza points out that without adequate care in the question how knowledge is managed, organizations will not be able to operate safely and optimally, and this can result in the ineffective and inefficient creation and delivery of products and services, which leads to unsatisfied customers that ultimately leads to the termination of the company (Desouza, 2011). The next reason for dealing with knowledge management is to help be different from competitors in both products, services and working culture. All organizations, whether for-profit or not-for-profit, compete within a given market. Knowledge management is a critical driver of competitive advantages because it increases the capacity of organizations to innovate. Organizations that are unable to innovate and grow capacities at a sustainable pace will lack the ability to continuously gain new clients, which can soon lead to their termination. Organizations that are able to innovate will be able to secure, and even retain, their competitive positions in the marketplace (Desouza, 2011). Globalization has also played its part in increasing the need for knowledge management because organizations wish to seek for effective supporting tools, methods and standardized processes for acquiring and sharing knowledge despite of many cultural hindrances, and to be able to come across countries and continents. The next important reason of expanding the necessity of knowledge management is aging labour labour force. Most organizations are experiencing a quick aging of their labour labour force and soon much knowledge will leave them due to fluctuation. This results in lack of intellectual capital which needs to be refilled, so that future generations in these working environments will not repeat the mistakes of the predecessors and reinvent the wheel again.

If an organization aims to be really conscious concerning knowledge management, it has to understand how important it is to effectively implement knowledge management systems. This means enforcing a strong, lively connection between the old best practices 
within the organization and the actions taken by the members of the organization based on that information. From this point of view the increasing need for such abilities as creativity and innovation seems justified. Knowledge management is strictly attached to these skills and covers an important skill for anyone working in today's organizations. So for the safe and sustainable operation of organizations the labour labour force which is able to use its knowledge in the given context is of crucial importance because knowledge has become probably the most important resource. There is still a debate on the question whether knowledge can be considered as a resource or not because unlike other traditional resources like capital or land, it is not scarce, but on the contrary, an abundance of shallow knowledge appears. So it is not the quantity or the availability but the quality and validity of knowledge that is questionable. The purpose of business organizations is to provide labour force for their operation, which is able to respond quickly to the challenges of the dynamically changing environment and the atypical, unexpected changes and effects. Constantly evolving employees are capable of building the learning organization of the F-Era, which achieves sustainability through continuously providing knowledge, new ideas and innovation.

Previously the significance of an effective, strategically approached system of knowledge management was presented, but from this aspect the quality of both personal and organizational knowledge is in focus. As it was discussed, personal, tacit knowledge of the individuals first must be made explicit knowledge, and then it has to be available for the appropriate members of the organization. The difficulty resides in the different capabilities of individuals in applying their obtained knowledge under specific working conditions and situations, which require different ways of externalizing their knowledge. If an employee is not able to rephrase and re-contextualize its knowledge according to the new, previously unknown circumstances, they will not contribute to the successful operation of the knowledge sharing system. So it seems that the biggest challenge for educational institutions is the question how can they train latter employees who can perform in a fast paced working environment where their knowledge becomes out of date as quickly as lightning, where the knowledge of yesterday is insufficient and the knowledge of today is sufficient only at best. The knowledge of persons participating in traditional, academic education, the knowledge of subsequent leaders and decision makers of the future refers to the past, and is about 'know how'. Contrarily, most organizations are about to employ labour labour force which is able to apply their knowledge in a given working environment knowing the domain of validity and also knowing what 'there and then' can be applied from the knowledge they have. So the emphasis has been moved from 'know how' to 'know when'. So as it was discussed above, considering the four basic elements of knowledge management there is an increasing emphasis on people, and especially on the capabilities and competencies of people. It is obvious that a successful knowledge management system cannot work without precisely defined and built processes and that the support provided by information and communication technologies is inevitable, too, but knowledge in itself without the people capable for contextualizing it is worth basically nothing.

In the process of selection and recruitment of most organizations not only the qualifications and language skills but also personal and professional competences of applicants to be employed play a key role. There are (at least) two different definitions of competence in practice. In general, or the way human resource experts and managers use it, a person is competent if it is able to reach a certain level of performance or handle a situation or problem due to abilities and knowledge (Boyatzis, 2008). However, according to another viewpoint a person can be considered competent if it is able to 
contextualize and apply its knowledge in a certain situation. So in other way being competent equals knowing how to interpret knowledge (Baracskai and Velencei, 2004). 'Competence is not the same as knowledge and it is not even an entity but a dynamic relationship of three entities: the knower, the knowledge and the context' (Dörfler, 2012). Consequently, two different persons with the same knowledge and abilities on the same professional field can perform differently based on the context. Furthermore, it is also possible that the same person in two different environments cannot achieve the same level of performance so they are competent in one situation but they are not in another.

To shed more light on this by an example, let's imagine two fresh graduates with great results during traditional education as junior lawyers. They both have the same knowledge on the field of civil law but one of them will be very successful at a huge law firm whilst the other simply cannot stand its ground in such a fast paced working environment but can perform above the expectations at a small business company, and vice versa. Or if we take a look at the example used before with the employee in the position of a janitor, he can perform according to the expectations if he is familiar with all the circumstances of the situation like cleaning the corridor, but may be incompetent when he has to clean the office of the chief executive officer due to embarrassment. The key point in all these differences is the context and the lack of ability to adopt to quickly changing circumstances and expectations. This also explains why the studies on organizational culture were so popular lately because the characteristics of the organizations can have a really significant impact on the competence levels of employees. Therefore, it is easy to understand that different organizational cultures mean different challenges for employees.

\section{Cooperation between Educational Institutions and Business Organizations}

Based on the previous findings it can be said that an organization is capable of handling the radical changes of its environment only if it develops its ability to adapt and furthermore, it can come before changes, and influence its environment itself. That is the reason why organizations try to establish and operate educational institutions on their own because this way they can provide themselves competent labour force for their safe operation. During the last thirty years we could see many examples of corporate universities and strategic associations with universities or shared education. The purpose of schools as such is to disseminate the culture and identity of the organization, to foster the development of not only on the job skills but teach competences like leadership, creative thinking or problem solving (Szoboszlai et al., 2014). Corporate universities or other institutions under different names as academies or centers for excellence are often operated by business organizations as strategic partners for the development of human capital according to the expectations and goals of the funding organization. In this way the students are allowed to get familiar with the culture of their latter employer, which later can really ease socialization for new employees.

These internal educational branches of business organizations are located halfway between universities and centers of academic knowledge and the world of business organizations. The validity of knowledge offered by them is different from the validity of knowledge offered by universities because it is basically organization-specific where actual or latter labour force is trained based on the needs of the institution. Consequently, all corporate universities are unique and different so it seems that there is no standard definition for what a corporate university really is. Still it can be said that students participating in such education can build knowledge and ability for adaptation which 
cannot be applied in all organizations, only in ones with the same organizational culture this knowledge can be considered as valuable.

\section{Ways of Knowledge Increase in the F-Era}

Based on the researches of Szoboszlai et al., the ways of knowledge increase can be interpreted by achieving a transformation on the bride between the world of universities ('know how') and the world of corporations ('know when') (Szoboszlai et al., 2014). Many different methods can be used during the individuals' learning journey such as training, coaching or mentoring.

The role of the persons participating in educating and training the leaders of the future is also an interesting question. These teachers are not really teachers in the old sense of the term. They need a great variety of previous experience as leaders or experts not only in one specific field but have to able to apply and possess transdisciplinary approach. In most situations they cannot act as teachers because a new way of both teaching and learning has to be applied, which is more informal, person related and indirect. So a teacher of such a corporate university is more likely to be a trainer, coach and/or mentor.

When speaking of trainers at corporate universities we have to step back from the concept we usually know about trainers dealing with development of skills and abilities like assertive communication or conflict management. A trainer is a person who helps to understand why it is necessary to practice the contextualization of concepts. It is not worth preparing for a situation that cannot even be planned, it is rather useful to be able to quickly adapt to new circumstances and unexpected situations. A trainer can help to accept and learn to be comfortable with the uncomfortable and to be able to act in 'here and now'. Training in such institutions focus on helping the trainees to be able to create a situation in which they can contextualize their known concepts and elements of knowledge or even non-existing ideas. All these trainings are created to provide an opportunity for the participants to learn from not only the instructor/trainer but also from each other and themselves. To achieve these goals trainers can rely on many different tools, techniques and methods like role-plays, simulations, classroom, computer- or web based instructions.

Another approach in such an environment that works is coaching. Participants of training and educational programmes at business academies are often busy leaders themselves with little time to read and learn about the most recent concepts and knowledge of the gurus. In such a situation a coach can help them by selecting the relevant knowledge for them and during the coaching sessions they together can contextualize new ideas. The task and responsibility of the coach is to filter the most recent knowledge of the gurus and find out which ideas are advantageous for the coachee. Business experts sometimes need the coach to discuss both dilemmas and new ideas and concepts with them or because due to the uncertainty he cannot do this with his subordinates or other leaders of the organization.

Coaches are often invited by leaders to help with their different business issues. Such a case is when the leader would like to update his knowledge with relevant new ideas, or wants some fresh inputs from someone with the same way of thinking and understanding of the given broader picture. A coach can also be useful if he can support the coachee in understanding how to apply old knowledge in a new context. Another way of supporting the coachee in the process of knowledge increase is to point out the new connections among old terms and ideas by rearranging their known rules. Business 
leaders sometimes require their coaches to help them understand their own knowledge, which must be made transparent for themselves and for their colleagues. It is important to notice that a coach is not an advisor so he does not provide advice for the coachee but enables the person to realize how to use their knowledge in another, new context.

Unlikely coaches, mentors give guidance and advice for the mentored persons, so it is a more developmental relationship. Speaking of a corporate university, all students act as both mentors and mentees, and learning techniques include such simple tools as explaining concepts to each other, which is a practical application of constructivism theory at work. This way it makes the students clarify, elaborate on and otherwise reconceptualise material by teaching each other (King, 2002).

\section{Conclusions}

The F-Era requires new ways for both learning and teaching. Knowledge workers of this era are eager to build valuable knowledge and are willing to use whatever tools and help they get. By now shallow and not validated knowledge is accessible for everyone who is willing to learn but the responsibility of searching and finding validated knowledge and/or to contextualize new knowledge by ourselves is constantly increasing.

Knowledge management has become a well-known and respected method for strengthening the competitiveness of all organizations. By understanding the nature of personal and organizational knowledge new methods and approaches have emerged in knowledge increase. It seems that the focus has been removed from 'know how' and the future is more about 'know when'. Corporations are looking for leaders and competent labour labour force who knows how to contextualize their knowledge so they can make the operation of the organization of the F-Era effective and efficient.

An important upcoming question is about the support of both learners and teachers. How can a corporate university be managed so that it could provide competent labour labour force for the organization? How will the process of learning change in such an environment in the future? So there are still many questions to be answered concerning knowledge increase in the F-Era.

\section{References}

Anderson, Chris, 2008. The Long Tail: Why the Future of Business is Selling Less of More. New York: Hyperion.

Baloh, P., Desouza, K. C. and Paquette, S., 2011. The concept of knowledge. In: K. C. Desouzaand S. Paquette (Eds.), Knowledge Management: An Introduction. New York: NY: Neal-Schuman Publishers, Inc., pp. 35-71.

Baracskai, Z., Dörfler, V., Szendrey, J.,Velencei, J., 2014. What is the Difference? There was Always Lifelong Learning. International Scientific Journal of Management Information Systems, 9(2), pp. 3-9.

Baracskai, Z., Velencei, J., 2004. Követő nélkül nincs vezető. Budapest: Myrror Media.

Blackler, F., 1995. Knowledge, knowledge work, and organisations: An overview and interpretation. Organisation Studies 16(6), pp. 1021-1046.

Bosua, R. and Venkitachalam, K., 2013. Aligning strategies and processes in knowledge management: a framework. Journal of Knowledge Management, 17(3), pp. 331-346. 
Boyatzis, R.E., 2008, Competencies in the 21st century, Journal of Management Development, 25(7), pp. 5-12.

Carr, N., 2010. The Shallows: What the Internet is Doing to Our Brains. New York: W. W. Norton \& Company

Davenport, T. H. and Prusak L. 2000. Working knowledge: How organizations manage what they know. ACM: Ubiquity.

Desouza, K. C., 2011. An introduction to knowledge management. In: K. C. Desouza and S. Paquette (Eds.), Knowledge Management: An Introduction. New York: NY: Neal-Schuman Publishers, Inc., pp. 3-34.

Dörfler, V., 2012, In-Cloud Knowledge Platform for CU - Neverending (ReContextualization of Knowing. Paper presented at the 2nd Corporate Universities and Ac@demies Summit, Paris, France

Godin, Seth 2008. Tribes: We Need You to Lead Us. London: Penguin Publishing Group

Greiner, M.E., Bo“hmann, T and Krcmar, H. 2007. A strategy for knowledge management. Journal of Knowledge Management, 11(6) 3-15.

Hislop, D., 2010. Knowledge management as an ephemeral management fashion? Journal of Knowledge Management, 14(6), pp. 779-790.

Jeon, S., Kim, Y. Koh, J., 2011. An integrative model for knowledge sharing in communities-of-practice. Journal of Knowledge Management, 15(2), pp. 251-269.

King, A., 2002. Structuring Peer Interaction to Promote Higher-Order Thinking and Complex Learning in Cooperating Groups. Vol. 41. No. 1.Promoting Thinking through Peer Learning. pp. 33-39.

Nonaka, I. Takeuchi, H., 1995. The knowledge creating company. New York, NY: Oxford University Press.

Oluikpe, P., 2012. Developing a corporate knowledge management strategy. Journal of Knowledge Management, 16(6), pp. 862-878.

Polanyi, M., 1962. Personal Knowledge: Towards a Post-Critical Philosophy. London: Routledge.

Polanyi, M., 1967. The tacit dimension. London, UK: Routledge and Kegan Paul.

Ponzi, L Koenig, M., 2002. Knowledge management: Another management fad? Information Research, 8 (1), paper no. 145.

Riege, A., 2007. Actions to overcome knowledge transfer barriers in MNCs. Journal of Knowledge Management, 11(1), 48-67.

Scarbrough, H. Swan, J., 2001. Explaining the diffusion of knowledge management. British Journal of Management, 12(1), pp. 3-12.

Serenko, A, Bontis, N., Booker, L, Sadeddin, K. Hardie, T., 2010. A scientometric analysis of knowledge management and intellectual capital academic literature (1994-2008). Journal of Knowledge Management, 14(1), pp. 3-23.

Strati, A., 2007. Sensible knowledge and practice-based learning. Management Learning, 38(1), pp. 61-77.

Szoboszlai, V., Velencei, J., Baracskai, Z., 2014. Post-Experiential Education: from Knowledge to 'Knowing'. Acta Polytechnica Hungarica, 11(10), pp: 235-247. 
Yakhlef, A., 2010. The corporeality of practice-based learning. Organisation Studies, 31(4), pp. 409-430 